\title{
Optimization of Lift Gas Allocation using Evolutionary Algorithms
}

\author{
Sofía López \\ University of Barcelona
}

Spain

\author{
Urhan Koç \\ Istanbul Polytechnic \\ Turkey \\ Javad Rahmani \\ Islamic Azad University \\ Iran
}

Emma Bakker

Leiden University

Netherlands

\begin{abstract}
In this paper, the particle swarm optimization (PSO) algorithm is proposed to solve the lift gas optimization problem in the crude oil production industry. Two evolutionary algorithms, genetic algorithm (GA) and PSO, are applied to optimize the gas distribution for oil lifting problem for a 6-well and a 56-well site. The performance plots of the gas intakes are estimated through the artificial neural network (ANN) method in MATLAB. Comparing the simulation results using the evolutionary optimization algorithms and the classical methods, proved the better performance and faster convergence of the evolutionary methods over the classical approaches. Moreover, the convergence rate of PSO is 13 times faster than GA's for this problem.
\end{abstract}

Keywords: particle swarm optimization; crude oil lifting; lift gas allocation; optimization; artificial neural network; genetic algorithm.

\section{INTRODUCTION}

There exist a wide variety of natural mechanisms to drive crude oil from the underground reservoirs to the surface, including the gas expansion and water pressure mechanisms. When the natural energies to produce crude oil from a well is not sufficient, the artificial lift procedures are used to accomplish the oil production process. In general, the artificial lift processes are divided into two main categories; gas-based lift process and pump-based lift process [1-8]. Gas-based lift technology is known as an efficient and economical procedure in the oil production industry. In a gas-based lift process, the optimal rate for the gas injection is determined such that it can compensate for the hydro-static pressure drop and frictional pressure drop in the well [9]. The optimum injection rate is important, mainly because of the operating constraints related to the available gas intake.

One of the very first studies on gas allocation optimization was conducted by Redden et al. in 1974 [10]. Authors in [10] have optimized the gas distribution among 30 wells in Venezuela. Their approach was based on the good laboratory practices (GLP) diagrams, and the optimization criterion was the higher profit rate. Their proposed strategy did not consider any optimization constraints; i.e., they assumed the unlimited amount of gas is available. A similar study is conducted by Mayhill in 1974 [11]. In 1981, Kanu and his colleagues introduced a parameter called the economic slope, which was a measure of the economic efficiency in a gas-based lift process. In their proposed approach, the optimal gas allocation was analyzed with and without constraints; e.g., with limited and unlimited gas intake [12]. In a further study, Nishikiori et al. developed a strategy based on the economic slope parameters, in which the optimum amount of gas injection was determined through a pseudo-Newtonian method [13]. In [14], authors optimized the controller tuning process using the particle swarm optimization. The objective of the optimization problem in their approach was to maximize the production rate. They also utilized GLP diagrams in their method. In another study, [15] developed a distributed algorithm to optimize the energy allocation in a building environment [15]. In [16], the rate of lift gas injection is determined based on the net present value (NPV). From their study, it is realized that the maximum profit from the production does not necessarily occur at maximum production. Authors also proved that the oil price is an important parameter in the optimization process, and an appropriate optimization scenario should be picked considering the oil price rate. However, the authors did not provide a well-designed model for their strategy. [17] applied the control theory principles to optimize the lift gas distribution; their approach was a cascaded control strategy. [18] developed an algorithm based on ant colony algorithm (known as continuous ant colony optimization, or CACO) to solve the gas allocation problem.

In this paper, the optimum amount of lift gas is distributed over a set of wells based on an evolutionary optimization algorithm. It is the first time that the particle swarm optimization (PSO) algorithm is used for finding the optimal gas injection rate for oil lift process. Worth mentioning that PSO algorithm is known to be more efficient and faster in solving such optimization problems, compared to the similar evolutionary algorithms such as genetic algorithm (GA). Moreover, in this study, the artificial neural network (ANN) method is utilized to estimate the performance plots of the gas-based lift process.

The rest of the paper is organized as follows. The next section explains the two evolutionary algorithms; genetic algorithm (GA) and particle swarm optimization (PSO). Section 3 describes the PSO algorithm challenges. The proposed strategy is shown in section 4 . Section 5 includes the simulation results. The work finishes with the conclusions in section 6 . 


\section{EVOLUTIONARY ALGORITHMS}

In this section, the genetic optimization algorithm (GA) and the particle swarm optimization (PSO) algorithm are explained in detail.

\subsection{Genetic algorithm}

Genetic algorithm is one of the most important meta-heuristic algorithms, first introduced by Holland in 1975 [19]. Genetic algorithm is a type of evolutionary algorithm, which is commonly used in artificial intelligence (AI) and computing. The genetic algorithm applies a set of solutions to the optimization problem in each generation. The selection process chooses the individuals with the best fitness; these individuals mutate and reproduce new genes [20-26]. Therefore, the best optimum solutions are attained through mimicking the natural process genes mutation, selection, and reproduction. In the genetic algorithm, the final goal of selections and mutations is to maximize the fitness or minimize the costs of each individual. The genes adapt themselves to the environmental conditions such that they survive or mutate with genes with higher fitness. The crossover operator is used to produce new offsprings from every two parents.

\subsection{Particle swarm optimization algorithm}

Extensive studies have investigated the social behavior of various types of creatures; such as birds flock, school of whales, fish, sharks, etc. The particle swarm optimization (PSO) algorithm is a meta-heuristic computational method that mimics the social behavior of animal swarms. PSO optimizes problem by improving the candidate solution iteratively. The algorithm was first introduced by Kennedy and Eberhart in 1995 [27]. Swarm intelligence is the collective behavior of self-organized systems. The algorithms in artificial intelligence (AI) follow a hierarchy directly or indirectly. In PSO algorithm, two main parameters are being updated in each iteration; velocity term and position term. The particle's velocity and position are updated through the following equations, respectively.

$v_{i}(t+1)=w v_{i}(t)+c_{1} r_{1}\left(y_{i}(t)-x_{i}(t)\right)+c_{2} r_{2}\left(\hat{y}(t)-x_{i}(t)\right)$

$$
\begin{aligned}
x_{i}(t+1) & =x_{i}(t)+v_{i}(t+1) \\
x_{i}(t) & \in U\left[x_{\min }, x_{\max }\right]
\end{aligned}
$$

where $v i(t)$ and $x i(t)$ denote the velocity and position of particles at time $t$. $y$ and parameters represent the personal best solution of the particle and the global best solution, respectively. $\mathrm{r} 1$ and $\mathrm{r} 2$ are the random vectors with uniform distribution in the $[0,1]$ interval. $\mathrm{w}, \mathrm{c} 1$, and $\mathrm{c} 2$ are the inertia coefficient, personal learning coefficient, and collective learning coefficient, respectively.

Beside the velocity and position updates, the personal best and global best parameters should also be updated in a standard PSO algorithm.

$$
\begin{aligned}
y_{i}(t+1) & =y_{i}(t) \quad f o r f\left(x_{i}(t+1)\right) \geq f\left(y_{i}(t)\right) \\
y_{i}(t+1) & =x_{i}(t+1) \quad \operatorname{forf}\left(x_{i}(t+1)\right) \leq f\left(y_{i}(t)\right) \\
\hat{y}(t) & =y_{0}, y_{1}, \ldots, y_{z}=\operatorname{minf}\left(y_{0}(t)\right), f\left(y_{1}(t)\right), \ldots, f\left(y_{z}(t)\right)
\end{aligned}
$$

The PSO algorithm is as follows.

- For each particle $i \in 1, \ldots, s$, initialize th position $x_{i}$ and velocity $v_{i}$ randomly.

- Set $y_{i}=x_{i}$

- For each particle $i$, evaluate the fitness function $f\left(x_{i}\right)$.

- For each particle $i$, update $y_{i}$ and $\hat{y}_{i}$ from (3).

- For each dimension $j \in 1, \ldots, N_{d}$, update the velocity from:

$$
\begin{aligned}
v_{i, j}(t+1) & =w v_{i, j}(t)+c_{1} r_{1, j}\left(y_{i, j}(t)-x_{i, j}(t)\right) \\
& +c_{2} r_{2, j}\left(\hat{y}_{j}(t)-x_{i, j}(t)\right)
\end{aligned}
$$

- Apply the position update to each particle.

- Stop the algorithm when the convergence criteria is met, otherwise, go to step 3 .

\section{PARTICLE SWARM OPTIMIZATION ALGORITHM CHALLENGES}

The particle swarm optimization algorithm has several drawbacks and disadvantages. PSO can easily fall into the local optimum points in high-dimensional optimization problems. Although PSO is faster compared to similar evolutionary algorithms, its convergence rate does not enhance with a higher number of iterations. The prominent reason is that in this algorithm, particles converge to the point with the personal best and global best solution. To address this issue, the inertia weight $\mathrm{w}$ is used to modify the algorithm [28]. Another main drawback in this algorithm is that the quality of solutions is very much dependent to the weighting coefficients and algorithm parameters [29]. Therefore, we should try to tune the PSO parameters in the best way.

\section{PROPOSED STRATEGY}

In order to define the optimization problem, we first need to estimate the performance diagrams of the wells with different levels of gas injections. The artificial neural network (ANN) algorithm is utilized in this step to attain the (good laboratory practices) GLP-based performance diagrams. The training model is then used as the fitness function in the optimization process. Once the convergence criteria are met, the algorithm stops. The PSO algorithm is simulated in MATLAB environment. The advantages of coding in MATLAB include: 
- The programmer can do the code testing, implementing, visualizing easily and fast without the need for sophisticated, time-consuming programming.

- MATLAB includes a large database of built-in algorithms and libraries. It also includes various embedded functions and tools; such as linear algebra function, neural network tools, probability functions, etc.

- The programmer can utilize the advanced programming techniques and object-oriented programming.

- The programmer can easily integrate MATLAB with other programming languages, or software. Also, the programmer is able to export to or import in any files between MATLAB and other software.

\section{SIMULATION RESULTS}

In this section, the results of gas allocation optimization using PSO algorithm are presented and discussed. Two different scenarios; a low-dimension problem with six wells, and a high-dimension problem with 56 wells are considered in our simulations. The constraints on the amount of available lift gas are considered (limited amount of lift gas is available). The optimization is implemented on the datasets from Buitrago et al. research. As mentioned, the ANN approach is employed to estimate the performance diagrams of the lift gas. The objective in the constrained optimization problem is to maximize oil production. The upper limit for the gas consumption is only considered as a constraint, and the gas consumption is not a term in the objective function. The objective function and the constraints equation is as (5).

$$
\begin{gathered}
\max Z=\sum_{i=1}^{\# \text { ofwells }} Q_{o_{i}} \\
\sum_{i=1}^{\text {\#ofwells }} Q_{g_{i}} \leq A G
\end{gathered}
$$

The simulation results for the six-well problem and 56-well problem, using the proposed approach and GA, are shown in Tables 1 and 2, respectively.

Moreover, the estimation of performance plots using the neural network approach are illustrated in Figure 1.

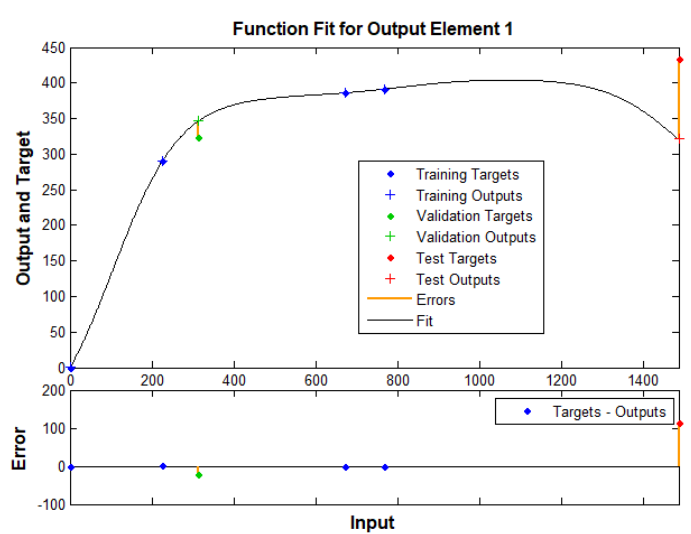

Figure 1: The performance plot estimates through the ANN algorithm.
Table 1: Simulation results on a 6-well problem using the propose method and GA

\begin{tabular}{|c|c|c|}
\hline Well 1 & $q_{g}(M S C F / D)$ & $q_{0}(B / D)$ \\
\hline PSO & 296.6 & 335 \\
GA & 296.6 & 335 \\
\hline \hline Well 2 & $q_{g}(M S C F / D)$ & $q_{0}(B / D)$ \\
\hline PSO & 507.7 & 720 \\
GA & 507.7 & 720 \\
\hline \hline Well 3 & $q_{g}(M S C F / D)$ & $q_{0}(B / D)$ \\
\hline PSO & 931.7 & 1079 \\
GA & 934.8 & 1079 \\
\hline \hline Well 4 & $q_{g}(M S C F / D)$ & $q_{0}(B / D)$ \\
\hline PSO & 353.9 & 534 \\
GA & 353.9 & 534 \\
\hline \hline Well 5 & $q_{g}(M S C F / D)$ & $q_{0}(B / D)$ \\
\hline PSO & 910.0 & 757 \\
GA & 910.0 & 757 \\
\hline \hline Well 6 & $q_{g}(M S C F / D)$ & $q_{0}(B / D)$ \\
\hline PSO & 3000.0 & 3425 \\
GA & 3000.0 & 3425 \\
\hline \hline
\end{tabular}

Table 2: Simulation results on a 56-well problem using the propose method and GA

\begin{tabular}{|c|c|c|}
\hline & $q_{g}(M S C F / D)$ & $q_{0}(B / D)$ \\
\hline PSO & 22500 & 22541 \\
GA & 22500 & 22541 \\
\hline \hline
\end{tabular}

From Table 1, the optimum oil production is 3425 barrels in the constrained optimization problem, in both PSO and GA approaches. In a 6-well optimization problem, the results from the two evolutionary algorithms GA and PSO is almost the same, since it is a low-dimension problem. Obviously, in a higher dimension optimization problem with more computational complications, the performance of the evolutionary optimization methods will be recognizably different. Comparing the results of simulations in a 56-well problem proved that the proposed evolutionary algorithms performed more than $3 \%$ (more than 700 barrels) better than the classic approaches. Therefore, if the higher the dimension of the problem, the significantly better performance will be attained using the evolutionary optimization algorithms compared to the classical methods.

Although the results from GA and PSO approaches are the same, we recommend PSO for the gas allocation optimization problem. To prove the superiority of PSO over GA, we have shown the number of iterations needed to solve the same problem using the two algorithms (Figure 2). Thus, from the iteration graph, PSO converges a lot faster (13 times faster) than GA and requires less number of iterations for solving the same optimization problem. So, the operational costs for solving the problem using GA is significantly more than the cost associated with PSO. The parameter update processes in 
PSO enhances the convergence pace in the algorithm. The main drawback of GA in this regard is that it does not update its parameters, and it does not include any tunable parameter in its process.
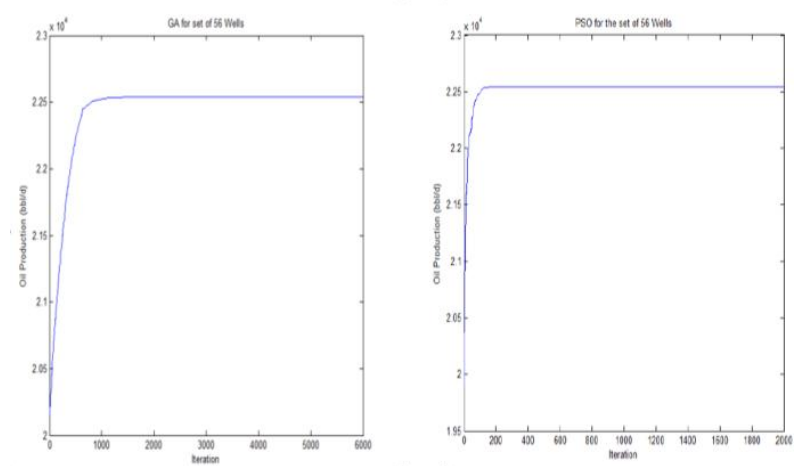

Figure 2: The number of iterations in PSO and GA for solving the 56-well problem

\section{CONCLUSIONS}

The gas distribution optimization problem is studied in this paper. The particle swarm optimization (PSO) approach is used for the first time for this problem. The performance plots are attained through an artificial neural network (ANN) learning. The proposed strategy is implemented on a highdimensional (56-well) and a low-dimensional (6-well) problem. The better performance of the evolutionary optimization method (GA and PSO) over the classical approaches is more recognizable when the problem is of higher dimension (like the 56-well problem). PSO and GA showed similar performances; however, PSO performed much faster (13 times faster) and required less number of iterations than GA.

\section{REFERENCES}

[1] F. Rahmani, F. Razaghian, and A. Kashaninia, "High Power Two-Stage Class-AB/J Power Amplifier with High Gain and Efficiency," 2014.

[2] M. Ketabdar, "Numerical and Empirical Studies on the Hydraulic Conditions of 90 degree converged Bend with Intake," International Journal of Science and Engineering Applications, vol. 5, pp. 441-444, 2016.

[3] A. Hamedi, M. Ketabdar, M. Fesharaki, and A. Mansoori, "Nappe Flow Regime Energy Loss in Stepped Chutes Equipped with Reverse Inclined Steps: Experimental Development," Florida Civil Engineering Journal, vol. 2, pp. 28-37, 2016.

[4] R. Eini and A. R. Noei, "Identification of Singular Systems under Strong Equivalency," International Journal of Control Science and Engineering, vol. 3, pp. 73-80, 2013.

[5] Rostaghi-Chalaki, Mojtaba, A. Shayegani-Akmal, and H. Mohseni. "Harmonic analysis of leakage current of silicon rubber insulators in clean-fog and salt-fog." 18th International Symposium on High Voltage Engineering. 2013.
[6] Rahimikelarijani, Behnam, et al. "Optimal Ship Channel Closure Scheduling for a Bridge Construction." IIE Annual Conference. Proceedings. Institute of Industrial and Systems Engineers (IISE), 2017.

[7] F. Rahmani, F. Razaghian, and A. Kashaninia, "Novel Approach to Design of a Class-EJ Power Amplifier Using High Power Technology," World Academy of Science, Engineering and Technology, International Journal of Electrical, Computer, Energetic, Electronic and Communication Engineering, vol. 9, pp. 541-546, 2015.

[8] Rostaghi-Chalaki, Mojtaba, A. Shayegani-Akmal, and H. Mohseni. "A study on the relation between leakage current and specific creepage distance." 18th International Symposium on High Voltage Engineering (ISH 2013). 2013.

[9] M. Golan and C. H. Whitson, Well Performance, Norwegian University of Science and Technology (NTNU), Trondheim, Norway, (1991) by PrenticeHall. Inc.

[10] J. Redden, T. A. Sherman, and J. Blann, "Optimizing Gas-Lift Systems," in Proceedings of Fall Meeting of the Society of Petroleum Engineers of AIME, 1974.

[11] T. D. Mayhill, "Simplified Method for Gas-Lift Well Problem identification and Diagnosis," in Fall Meeting of the Society of Petroleum Engineers of AIME, 1974.

[12] E. Kanu, J. Mach, and K. Brown, "Economic Approach to Oil Production and Gas Allocation in Continuous Gas Lift (includes associated papers 10858 and 10865)," J. Pet. Technol., vol. 33, no. 10, pp. 1,887-1,892, Oct. 1981.

[13] N. Nishikiori, R. A. Redner, D. R. Doty, and Z. Schmidt, "An Improved Method for Gas Lift Allocation Optimization," in Proceedings of SPE Annual Technical Conference and Exhibition, 1989.

[14] R. Eini, "Flexible Beam Robust Loop Shaping Controller Design Using Particle Swarm Optimization," Journal of Advances in Computer Research, vol. 5, pp. 55-67, 2014.

[15] R. Eini, and S. Abdelwahed. "Distributed Model Predictive Control Based on Goal Coordination for Multi-Zone Building Temperature." In 2019 IEEE Green Technologies Conference (GreenTech), Lafayette, LA. 2019.

[16] B. T. Hyman, Z. Alisha, S. Gordon, "Secure Controls for Smart Cities; Applications in Intelligent Transportation Systems and Smart Buildings," International Journal of Science and Engineering Applications, vol. 8, pp. 167-171, 2019. doi: 10.7753/IJSEA0806.1004

[17] Heng, Li Jun, and Abesh Rahman. "Designing a robust controller for a missile autopilot based on Loop shaping approach." arXiv preprint arXiv:1905.00958 (2019)

[18] Patel, Dev, Li Jun Heng, Abesh Rahman, and Deepika Bharti Singh. "Servo Actuating System Control Using Optimal Fuzzy Approach Based on Particle Swarm Optimization." arXiv preprint arXiv:1809.04125 (2018). 
[19] J. H. Holland, Adaptation in natural and artificial systems: an introductory analysis with applications to biology, control, and artificial intelligence. University of Michigan Press, 1975.

[20] Bakker, V. Deljou, and J. Rahmani, "Optimal Placement of Capacitor Bank in Reorganized Distribution Networks Using Genetic Algorithm," International Journal of Computer Applications Technology and Research (IJCATR), vol. 8, pp. 23198656, 2019.

[21] F. Rahmani, "Electric Vehicle Charger based on DC/DC Converter Topology," International Journal of Engineering Science, vol. 18879, 2018.

[22] F. Rahmani and M. Barzegaran, "Dynamic wireless power charging of electric vehicles using optimal placement of transmitters," in 2016 IEEE Conference on Electromagnetic Field Computation (CEFC), 2016, pp. 1-1.

[23] M. Ketabdar and A. Hamedi, "Intake Angle Optimization in 90-degree Converged Bends in the Presence of Floating Wooden Debris: Experimental Development," Florida Civ. Eng. J, vol. 2, pp. 2227.2016, 2016.

[24] M. Ketabdar, A. K. Moghaddam, S. A. Ahmadian, P. Hoseini, and M. Pishdadakhgari, "Experimental Survey of Energy Dissipation in Nappe Flow Regime in Stepped Spillway Equipped with Inclined Steps and Sill," International Journal of Research and Engineering, vol. 4, pp. 161-165, 2017

[25] A. Hamedi and M. Ketabdar, "Energy Loss Estimation and Flow Simulation in the skimming flow Regime of Stepped Spillways with Inclined Steps and End Sill: A Numerical Model," International Journal of Science and Engineering Applications, vol. 5, pp. 399-407, 2016.

[26] Rahimikelarijani, Behnam, Mohammad SaidiMehrabad, and Farnaz Barzinpour. "A mathematical model for multiple-load AGVs in Tandem layout." Journal of Optimization in Industrial Engineering (2019).

[27] J. Kennedy and R. Eberhart, "Particle swarm optimization," in Proceedings of ICNN'95 International Conference on Neural Networks, 1995, vol. 4, pp. 1942-1948.

[28] N. Sfeir, H. Sharifi, "Internet of Things Solutions in Smart Cities," doi: 10.13140/RG.2.2.26015.51367 August 2019.

[29] H. Sharifi, "Singular Identification of a Constrained Rigid Robot," International Research Journal of Engineering and Technology (IRJET), vol. 5, pp. 941946, 2018 